\title{
Mapping cityscapes into cyberspace for visualization
}

\author{
By Jiang Yu Zheng* and Min Shi
}

This work establishes a cyberspace of a real urban area for visiting on the Internet. By registering entire scenes along every street and at many locations, viewers can visually travel around and find their destinations in cyberspace. The issues we discuss here are mapping of a large-scale area to image domains in a small amount of data, and effective display of the captured scenes for various applications. Route Panoramas captured along streets and panoramic views captured at widely opening sites are associated to a city map to provide navigation functions. This paper focuses on the properties of our extended images - route panorama, addressing the archiving process applied to an urban area, an environment developed to transmit image data as streaming media, and display for scene traversing on the WWW in real time. The created cyberspaces of urban areas have broad applications such as city tour, real estate searching, e-commerce, heritage preservation, urban planning and construction, and vehicle navigation. Copyright (C) 2005 John Wiley $\mathcal{E}$ Sons, Ltd.

Received: 9 April 2004; Accepted: 7 July 2004

KEY WORDS: route panorama; panoramic view; cyberspace; streaming media; virtual tour; visualization; georeference

\section{Introduction}

A cyber city could either be a computer-generated 3D space as some fiction movies display, or a duplicate of a real geographical city with some virtual functions applied to it. The latter is normally more difficult because it has to acquire data faithfully from the real world. Recent multimedia and VR techniques have provided maps, images, video clips and 3D VRML models to represent real cities. However, the graphics-generated models lack reality. Even the model surfaces are mapped with textures for gaining the realism, and generating an entire area requires much laborious modelling by using interactive software. Another approach is to use real images. However, if the images are in coarse, sparse and discrete formats, people who have no knowledge of an area still feel difficulty in establishing the entire space in their mind from images taken from limited locations. This work investigates full image mapping of scenes from a

*Correspondence to: Jiang Yu Zheng, Department of Computer and Information Science, Indiana University-Purdue University, Indianapolis, IN 46202, USA. E-mail: jzheng@cs.iupui.edu large-scale urban area to cyberspace and seamless traversing of the area on the Internet.

Maps (aerial images) and images (including extended format, e.g. panoramic views) project scenes to planes orthogonally and to focal points in perspective projection, respectively. The linkage between maps and images has achieved a good style for space perception on the Web. However, a map has less detailed information visible on the ground. Discrete images may not cover global scenes sufficiently. To bridge global and local information more closely, this work creates another dimension of focus: it projects scenes towards lines along streets, which is achieved by dynamic scanning of scenes with a camera moving along the paths. It generates an extended image format called route panor$a m a$, which provides continuous views of the streets in a non-redundant way.

Streets are important components of a city not only because they connect geospatial locations, but also because they contain rich visual context closely related to our lifestyle and reflect human civilization. The established street models in the cyber cities can facilitate a broad range of applications such as finding an address, cyber tour, e-commerce, virtual heritage sites, 
urban planning and renewing and traffic navigation. The objectives of this work are to:

1. Design a scheme to capture scenes of interest in various image formats including route panoramas, panoramic views and around-object images. We scan all streets in an urban area to build an image-based city model.

2. Introduce the route panorama and its properties for mapping cityscapes to a grid of visual maps. We will focus on the projection, generated 2D shape and visibility of the route panoramas.

3. Develop a pseudo-3D display tool to transmit and display route panoramas on the Internet, linked from a map. Viewers will be able to traverse the streets interactively, look around, zoom in and out, and turn from one street to another.

The related works so far include panoramic views that project $360^{\circ}$ scenes toward static points obtained either through slit scanning while the camera is rotating $^{1,2}$ or mosaicking (photo stitching). ${ }^{3}$ The panoramic views are particularly representative at wide and open spaces. The early version of the route panorama is called generalized panoramic views (GPV), first invented for mobile robot navigation. ${ }^{1,4,5}$ The GPV is a special case of a more general image representation called dynamic projection image, ${ }^{6}$ which comprise many slit views taken at different time instances when the camera moves along a path or a static camera looks at a dynamic flow. On the display aspect, $\mathrm{Li}^{7}$ has associated the generated panoramic views with a global positioning system (GPS) for navigation in an urban area. This topic was also expanded ${ }^{8,9}$ from slit scanning to the stripe mosaic using image patches, which requires feature correspondence between consecutive patches. Although they eventually generate a nice $2 \mathrm{D}$ view, the computationally expensive matching algorithm between consecutive frames may suffer from occlusion and limits the extendibility to long routes.

\section{Mapping Cityscapes to Cyberspace}

The goal of this work is to map all street scenes to a cyberspace using various types of images for city traversing and indexing. The criteria of cityscape mapping are as follows:

- Complete: mapped scenes should include the entire area in a city, covering the landscapes and architectures as much as possible.

- Continuous: visual data should be seamlessly connected and easy for streaming data transmission and display over the Internet, which allows viewers to travel from place to place in the cyber city continuously.

- Compact: images should have less redundant coverage of scenes so that they minimize storage and transmission bandwidth, and give fast responses.

We start from a map (Figure 1) to project a real city to images. As commonly displayed on the Internet, many locations have links to discrete images showing scenes there. In the case where many scenes around can be observed from one location, e.g. park, square or outlook, a panoramic image can be taken to include scenes at all
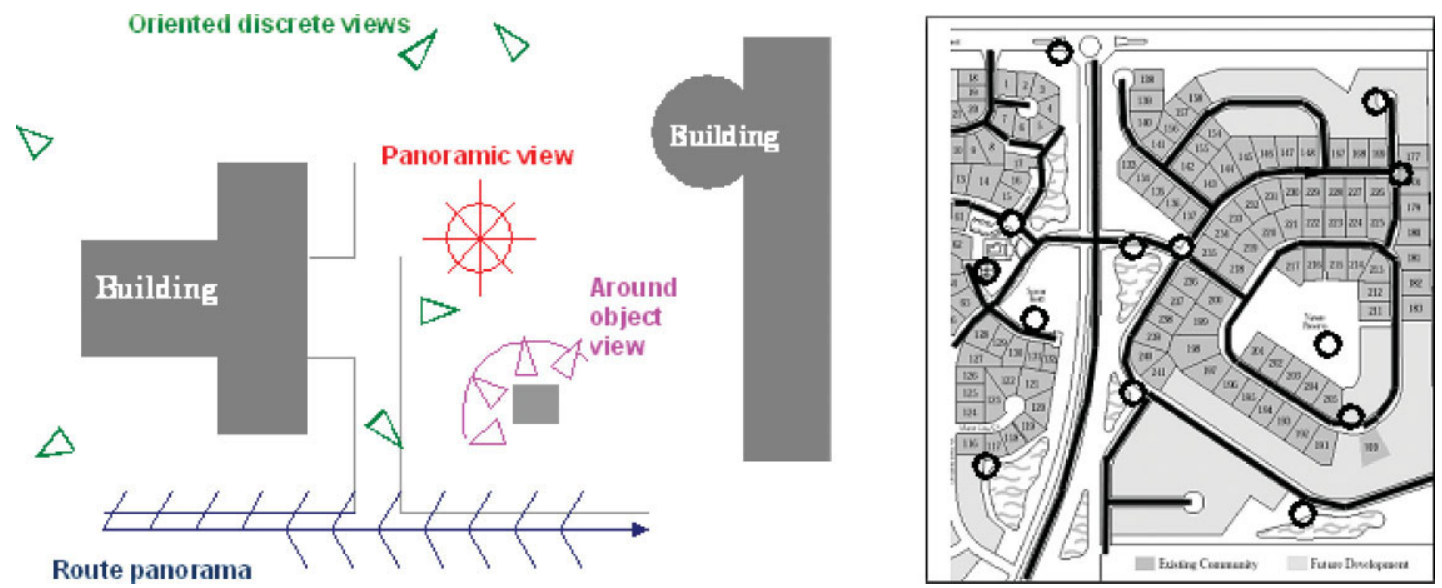

Figure 1. Various projections covering landscapes in a map. (a) Different types of images taken at various locations. (b) Route panorama and panoramic view covered scenes along every road and at circled positions respectively. 


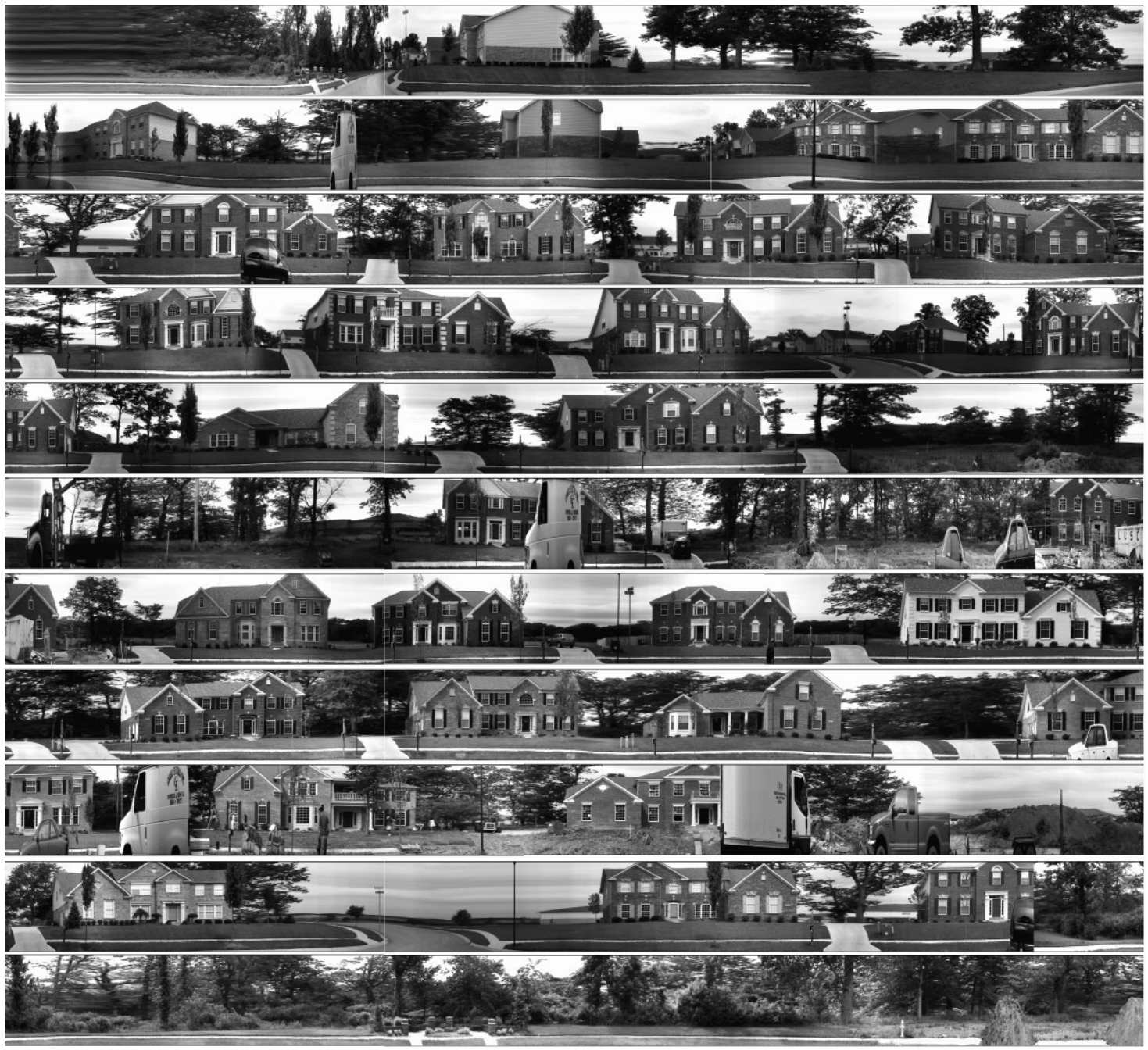

Figure 2. Route panorama from one side of a street.

orientations. Scenes around the focal point are then mapped onto a cylindrical image surface, a conic image surface (for high architectures) ${ }^{6}$ or a spherical surface. Among them, we separate local and global panoramas. A local panoramic image contains close scenes, while a global panoramic view contains remarkable and distant scenes visible in the entire area. Mountains and city skylines viewed from an open space or on top of a building can be considered a global panorama.

If an architecture or object has rich visual context on each side, several discrete images may be taken at selective distances and orientations to cover all its aspects. This is suitable for observing monuments, sculptures or houses. If the images are densely taken, we obtain around-object views of the object.
In this work, we add a new mapping of cityscapes: route panoramas. ${ }^{10}$ We project scenes on one side of a street towards a smooth path along the street, which may be a curved one, on the horizontal plane. A route panorama is created by scanning route scenes continuously with a virtual slit camera that substantially picks up a pixel line in the image frame. The connection of pixel lines from the consecutive images forms a long, continuous 2D image belt containing major scenes of the street. An example of a route panorama is given in Figure 2.

The panoramic view has a data size smaller than the image sequence turning around at the same position because the redundant data in the overlapped images is dropped. Similarly, a route panorama saves much data 
compared with a sequence of discrete images (or video) covering the same scenes along the route. Ideally, if the image frame has a width $w$, the route panorama only has $1 / w$ of the data size of the entire video sequence, since we only extract one pixel line from each frame when viewing through a slit. This shows a promising property of the route panorama as a visual index, which can deliver large amounts of information with minimal data. The route panorama allows a full registration of scenes along streets. Driving a vehicle through streets may increase the modelling area of the city significantly.

\section{Acquiring Complete Route Panoramas along Streets in a City}

\section{Scanning Scenes from a Moving Vehicle}

We mount a camera on a vehicle that moves along a smooth curve on a horizontal plane. A car, bus, train, or boat can produce such a camera motion that has one degree of rotation around the vertical axis and a translation in the path tangent direction. The camera path is described by $S(t)$, where $t$ is the time and is related to the horizontal axis in the route panorama. We divide a path roughly as linear, concave or convex segments depending on the sign of curvature. The vehicle speed is kept constant by a cruising system to yield the camera positions, or the path is recorded by GPS.

Extending from a simple slit setting in the camera frame facing sideways, ${ }^{10}$ we propose a projection of route panoramas for a flexible camera setting. Through our investigation, we find that the camera tilt determines the vertical coverage of the route panorama for a certain height of scenes. The slit location determines object aspects (front surfaces, or front and side surfaces) to capture along a route, and the position of the slit in the camera frame preserves a good shape of an object in the route panorama. Through a slit, the plane of sight, named plane of scanning (PoS), scans scenes during the camera motion as depicted in Figure 3. On the PoS, scenes are projected towards the camera focus through a lens, which is a thin perspective projection. The angle between the PoS and the motion vector $V$ of the camera is denoted by $\alpha(\alpha \neq 0)$ and is fixed after the camera is mounted. This angle determines the object aspects to be scanned along the route. By setting angle $\alpha$ of the PoS from the motion vector, we can obtain different aspect views of architectures or scenes. The more the angle

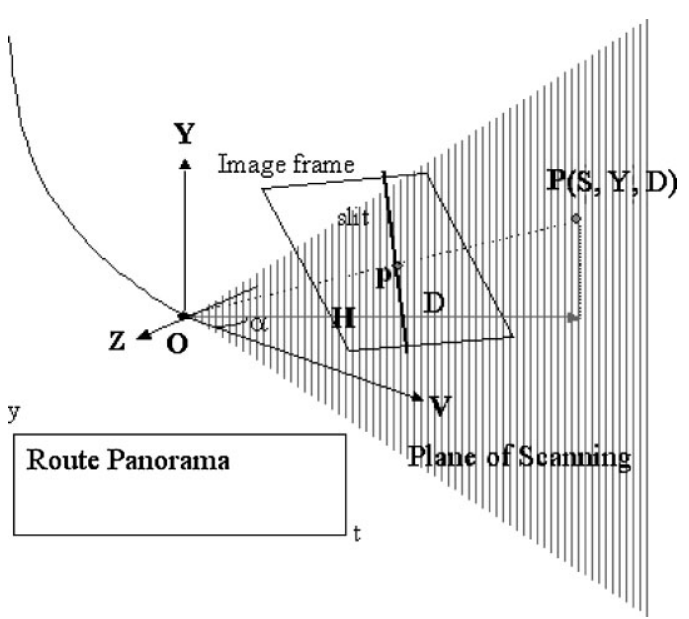

Figure 3. A PoS and a slit in scanning scenes.

deviates from sideways $(\alpha=\pi / 2)$, the longer the side views of architectures are captured in the route panorama.

There are many vertical lines on architectures, as the camera moves on a horizontal plane. If we select the PoS to be vertical in the $3 \mathrm{D}$ space in the scene scanning, we will obtain many good properties either for a linear or curved camera path. These properties will be discussed in the next section.

\section{Locating Slits in the Image Frame for Various Scenes}

After the direction of PoS is determined, the vehicle is able to move out with an approximate camera setting, which is flexible in the real situation. The vertical field of view of a route panorama is determined by the camera tilt. By directing the camera axis upward, we can capture high-rise buildings.

Now, we determine the virtual slit (pixel line) in the image frame. Locating a slit exactly at the projection of PoS will produce good shapes of objects in the route panoramas. According to the constraint of vertical PoS, $3 \mathrm{D}$ vertical lines are instantaneously scanned. This is invariant to the camera translation and rotation, and therefore invariant to the camera motion along a smooth path on the horizontal plane. The vertical lines in the $3 \mathrm{D}$ space are then guaranteed to be vertical in the route panorama. At any instance, the projections of 3D vertical lines have a vanishing point in the image plane if they are extended, according to the principle in computer vision. If the camera axis is horizontal, the vanishing 
point is at infinity. If we name the vertical axis through the camera focus by position axis of the camera, the vanishing point is thus the penetrating point of the position axis through the image plane. It is not difficult to prove that the slit, which is the intersection of the image plane and the vertical PoS, must go through the vanishing point.

In order to preserve shapes in route panoramas, we calculate the vanishing point and then locate the slit in the image passing through the point. After the video sequence is taken along a route, we select several arbitrary images from the sequence. Using edge detection to extract the projections of vertical lines in the images, a least squared error method is used to find the position of the vanishing point where all extracted lines cross each other. Passing the vanishing point, we locate a slit in the image frame and use it to scan the entire video sequence. Figure 4 shows an example of locating three slits (corresponding to three PoSs) in the image frame to obtain forward, side and backward route panoramas; all contain front surfaces, and forward and backward route panoramas contain side surfaces as well.

\section{Shapes in Projected Route Panoramas}

This section examines basic shapes of objects in the route panorama for display and street model recovery. Under the defined projection, scenes along a street are mapped onto an image belt or surface that is swept out with a pixel line $l$ along the path (Figure 5). The pixel line has a fixed relation with the path: the horizontal line
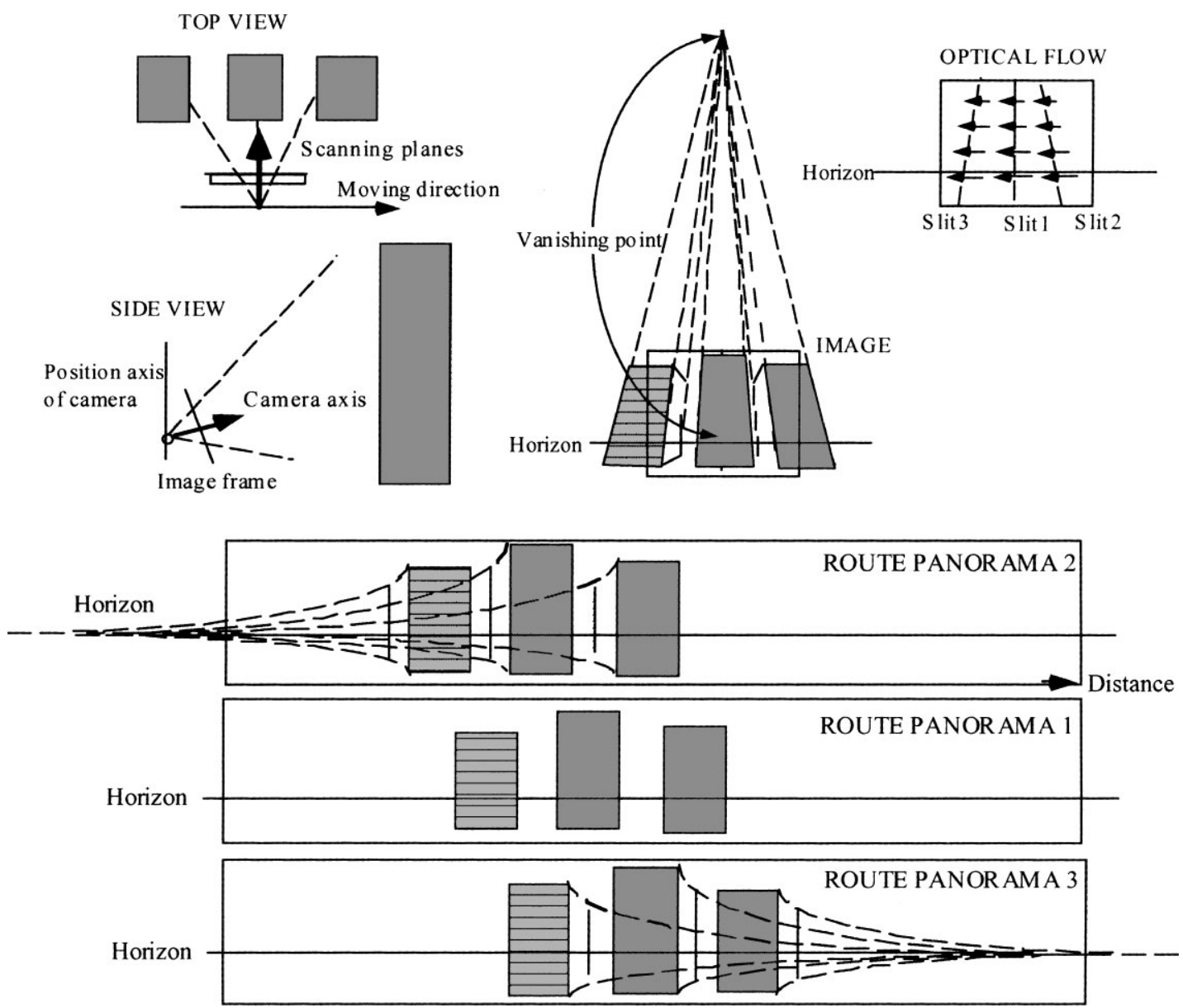

Figure 4. Route panoramas obtained by using an upward camera in a sideways direction. SIDE VIEW: the camera axis is directed upward higher than the horizon. IMAGE: vertical lines pass a vanishing point if they are extended. Horizon is lowered because of the upward camera direction. Slits are set passing through the vanishing point. ROUTE PANORAMAS: projected horizons are lower than the image centre. The horizontal parallel lines converge to the projected horizons as hyperbolas. 


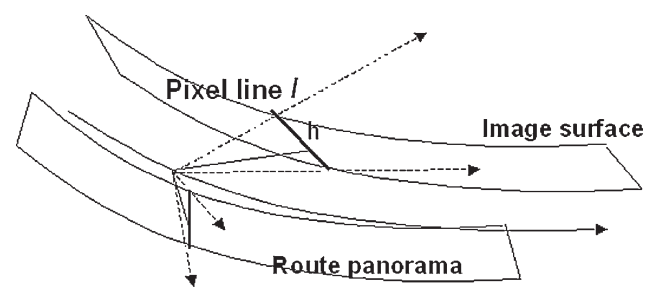

Figure 5. Projection of scenes on to route panoramas.

$h$ connecting $l$ from the camera focus on the path has a constant angle $\alpha$ with respect to the tangent direction of the path. The angle between $l$ and $h$ determines the vertical field of view of the route panorama. Because the route panorama is generated by perspective projection along the slit direction and locally parallel projection towards a smooth path, Table 1 summarizes the projections of the route panoramas for various $\alpha$.

We define the camera coordinate system $O-X Y Z$ where $\boldsymbol{O}$ is the camera focus, $\boldsymbol{X}$ is the moving direction and $Y$ is the vertical direction. Normally, the path of the camera is restricted within the street. Architectures are also constructed in parallel to the street. We focus on three types of structural lines parallel to the axes of system $\boldsymbol{O}-\boldsymbol{X Y Z}$ to investigate their shapes in the route panorama. Any other lines can be represented as a linear combination of these linear vectors. If we denote these lines by $\boldsymbol{A}, \boldsymbol{B}$ and $\boldsymbol{C}$, their projections in the route panorama from a linear path can be summarized as follows (Figure 6):

(i) A vertical line in the $3 \mathrm{D}$ space is scanned instantaneously and leaves its projection in the route panorama as a vertical line.

(ii) A line parallel to the camera motion vector is projected horizontally in the route panorama.

(iii) Other lines that cannot be described by the above lines are projected as hyperbolic curves in the route panorama.

It can be proved that the front surfaces of objects comprising lines $\boldsymbol{A}$ and $\boldsymbol{B}$ retain their shapes, since the

\begin{tabular}{|llll|}
\hline & $\alpha$ & Linear path & Curved path \\
\hline$\neq 0$ & $=\pi / 2$ & Orthogonal-perspective projection & Bended-orthogonal-perspective projection \\
& $\neq \pi / 2$ & Parallel-perspective projection & Bended-parallel-perspective projection \\
\hline
\end{tabular}

Table I. Projections of the route panorama according to the direction of PoS

a)

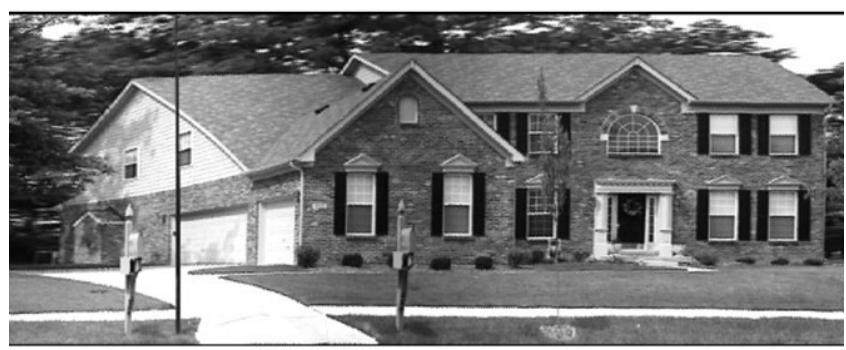

b)

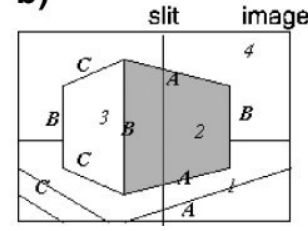

c)

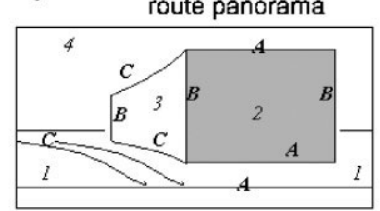

Figure 6. Typical lines and planes in the scenes along a street. (a) A perspective image taken in a forward direction. (b) A route panorama from the slit in the image (c) A section of real route panorama from a forward PoS in which both front and side surfaces are visible. 
vertical rims are still vertical and lines parallel to the road are still horizontal in the route panorama. The distortion on the aspect ratio is related to the camera moving speed and the surface depth. The scale of $A$ type lines is proportional to their real length in the 3D space. The vertical scaling on a $\boldsymbol{B}$ type line is from the perspective projection along the slit direction. The length of the $B$ line in the route panorama is inversely proportional to its depth. Unlike a perspective view, a small tree will never occlude an entire building in the route panorama, since the horizontal scale of an object is proportional to its real width along the road.

The difference of the route panorama from perspective projection is a 'curving' effect on lines in category $C$, which stretch in depth from the camera path. We can observe this effect in Figure 6, and further prove that $C$ type lines become hyperbolic curves. In the route panorama, the length of such a curve along the horizontal axis (the $t$ axis) is proportional to its length in the $3 \mathrm{D}$ space (the details are omitted here).

Another characteristic worth addressing is the convergence of parallel lines in category $C$. Under perspective projection, parallel lines with depth changes are not projected in parallel in the image frame. Their extensions cross at a vanishing point in the image plane. In a route panorama obtained from a linear camera path, however, parallel lines stretching in depth are projected to hyperbolic curves that have a common asymptotic line. Particularly, if the parallel lines are horizontal in the 3D space, their asymptotic line is the projection of horizon in the route panorama.

\section{Rendering Route Panoramas for Real-Time City Traversing}

\section{Streaming DataTransmission and Interactive Display of Streets}

When a route extends to many miles, downloading entire route panoramas and then displaying them as general graphics on a web browser is impossible owing to the shortage of memory on end computers and limited bandwidth on the mobile terminals. We display the acquired route panoramas for viewers to continuously traverse a city back and forth in large areas. Long route panoramas are transmitted as streaming data on the Internet and street scenes are seamlessly scrolled according to the viewer's interaction. The portability of the route panorama on a PDA or wireless phone also requires an efficient rendering algorithm. We have developed a progressive data transmission function that displays route panoramas during data downloading. A route panorama is segmented to many image sections in advance and indexed according to their locations. The sections ahead of the viewer's position are consecutively transmitted and connected in display. This gives viewers a quick response for free manoeuvring of routes.

Besides the simple display of route panoramas as a route profile (Figure 2) and a route image scroll that simulates one side view of a route, ${ }^{10}$ we develop a pseudo-3D display called panoramic traversing window on the WWW for virtual moving along a route. For a navigation task, a narrow perspective view may affect the perception of the spatial relations of scenes. We display a wide field of view in a panorama window.

For a traditional panoramic view, surrounding scenes are projected on to a cylindrical retina. Horizontal structure lines in the 3D space appear as sinusoidal curves in the opened retina surface and vertical lines in the 3D space stay vertical in it. A panoramic traversing window shows approximately a half circle $\left(180^{\circ}\right)$ of scenes in the viewer-selected direction. We can imagine that scenes in the route panoramas are first mapped onto walls on two sides of the street (Figure 7). If the path turns along a curved road based on the map, the walls are curved accordingly. The two walls are then projected on to a cylindrical image surface and we display the opened 2D form of it (Figure 8). A cylindrical screen can achieve an even better result.

A global panorama is prepared to present general orientation in a large area. It may contain distinct landmarks such as mountains and skylines. Although the global panorama is taken at a location different from the viewer's position during street traversing, it still gives an approximate orientation because of the scene distances in it.

The panoramic traversing window provides the following functions:

- Viewing around: the viewer can rotate smoothly by mouse clicking to view the street stretching forward, or view building fronts on either side of the street.

- Traversing: the viewer can translate along the street back and forth, while scenes move from one end of the street to the other in the field of view. At street crossings, the viewer can click to turn left or right to move on to another street.

- Field of view: the viewer can control the field of view by zooming in and out. This provides a switching 


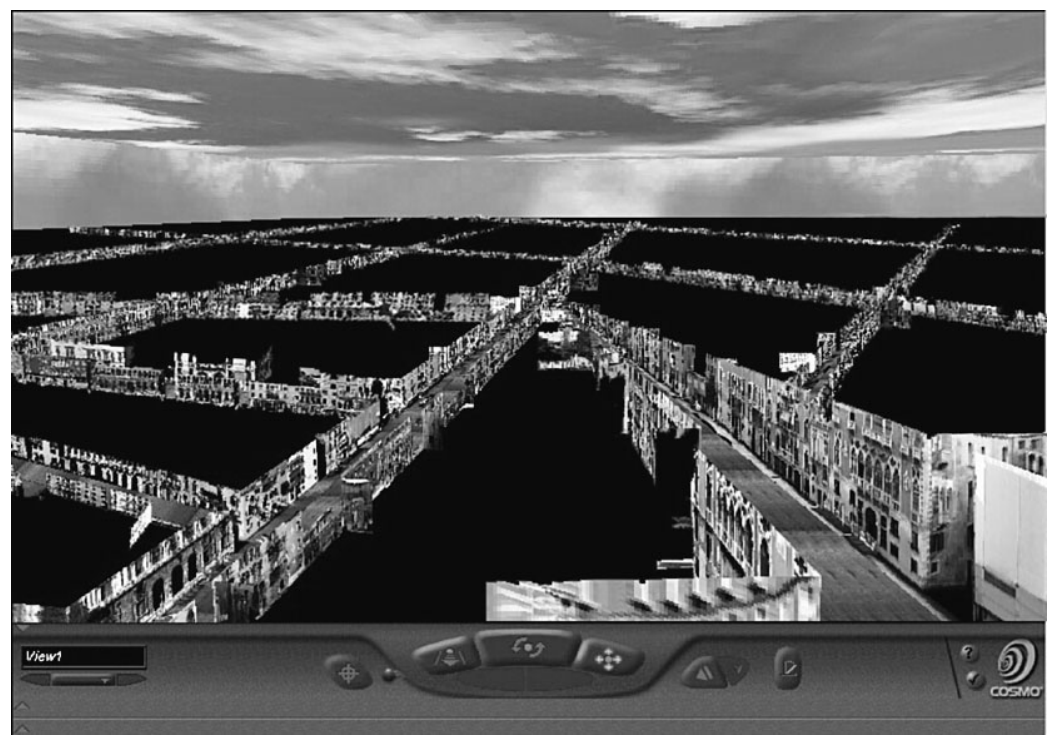

Figure 7. Every street is virtually bounded by two walls mapped with route panoramas.

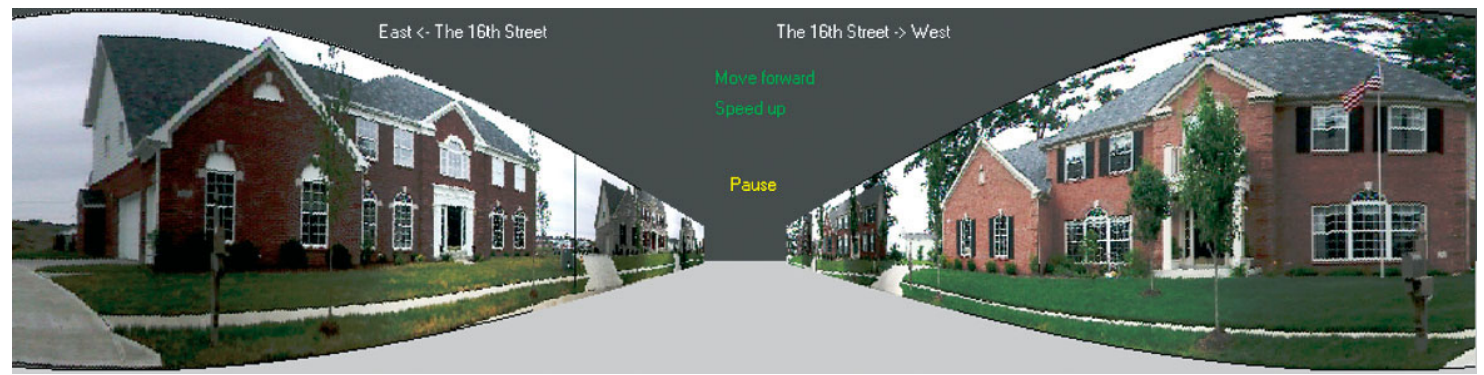

Figure 8. An opened form of the cylindrical surface dynamically displaying forward and side views.

between a wide panoramic view and a narrow perspective view.

\section{Rendering a PanoramicTraversing Window}

One preparation for rendering is to remove the sky area of the route panoramas (e.g. make it transparent in a GIF image) in order to see background scenes provided by the global panorama.

Route panoramas and the global panorama are aligned in the display according to their projected horizons. The height of the horizon in the panoramic traversing window is determined from that in the route panoramas through calculation of the vanishing point of vertical lines in the images (process omitted here). Calculation of the mapping from the route panorama to the traversing window is as follows. Assume the distance between the two street walls is $W$ and the horizon is at height $H$ in the route panorama. The camera path is characterized by travelling distance $S$. The route panoramas are mapped on to the walls and then projected towards the cylinder at the current position $S_{0}$. Assume a point $p(t, y)$ on the route panorama is mapped to $(\phi, \mu)$ in the panoramic traversing window, where $\phi \in[-180,180]$ is the spanned angle of view from the forward direction. The coordinates $(\phi, \mu)$ can be calculated by

$$
\phi= \pm \tan ^{-1} \frac{W / 2}{S-S_{0}} \quad \mu=\frac{Y \sin \phi}{W / 2}+H=y \sin \phi+H
$$

where $S \in\left[S_{0}-L, S_{0}+L\right]$ and $L$ is a short distance for scene rendering. According to the equations, rendering the traversing window is done by scaling and pasting vertical patches from the route panoramas (Figure 8). 


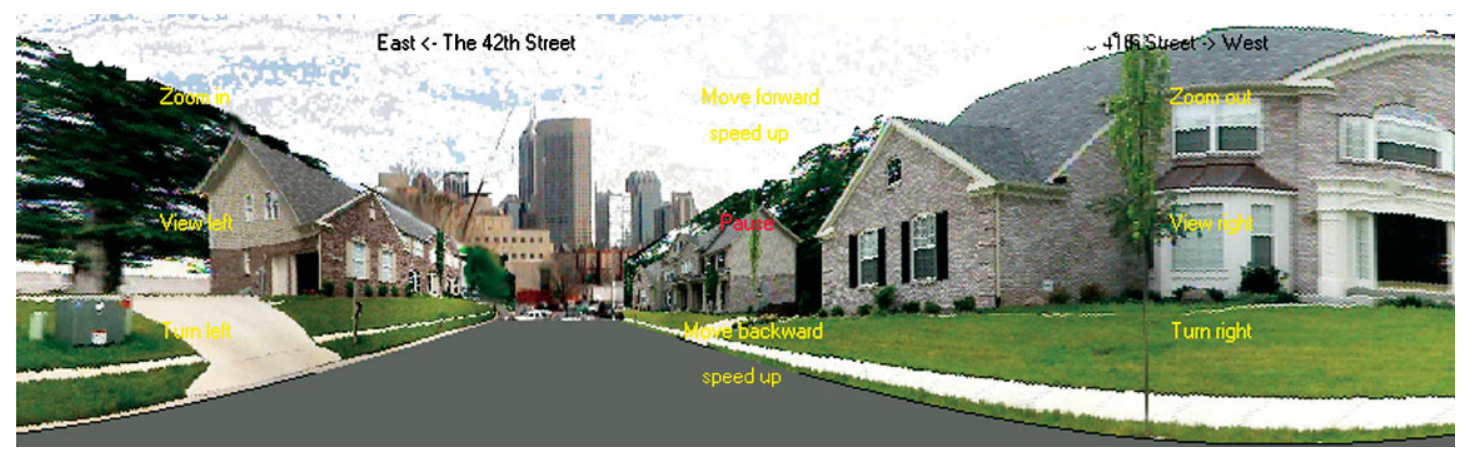

Figure 9. A panoramic traversing window dynamically displaying route scenes in an opened form of panoramic view. Traversing control is realized by mouse clicking in embedded sub-areas.

Although the traversing window is not a true 3D display, major portions in it have a similar motion to that in real 3D scenes.

The rendering processes are then as follows:

(i) The global panorama is mapped to an intermediate layer, on which the ground area lower than the horizon is further painted with a selected colour.

(ii) Above that, route panoramas are rendered in $360^{\circ}$ according to the viewer's location during the translation (Figure 9). This dynamic rendering generates optical flow along the sinusoidal curves, which is the same flow direction as a real translation appearing on the cylindrical panoramic view.

(iii) A view frame is copied from the intermediate layer to the browser. The view frame can be shifted horizontally and scaled with respect to its centre to change the orientation and the field of view, respectively. This generates optical flow equivalent to rotation and zooming. In the view frame, areas clickable by mouse are designed for the viewer's motion control including forward/ backward translation, viewing left/right, zooming in/out and turning left/right at the next street crossing.

(iv) Turning at a street crossing is visualized through three steps. When the viewer arrives at the street crossing, the view frame smoothly rotates towards the orthogonal direction of the current viewing direction. Meanwhile, the route panoramas of the next street are buffered and pasted in the intermediate layer. When the rotation finishes, the view frame switches $90^{\circ}$ back to the previous orientation in the intermediate layer, which realizes the transition to the second street.

\section{Viewing A Cyber City on the Internet}

We define visual node as a data set representing a space in the cyber city. Visual nodes can have different types, such as location $(0 D)$, route $(1 D)$, region $(2 D)$ and block $(3 D)$; each of them is displayed with a window consisting a panorama frame, a text frame, a map frame, and its relation with other visual nodes defined by menus (Figure 10b). We use Java for displaying visual nodes.

For various visual nodes, the panorama frame displays different views. A location is associated with a panoramic view. A route is covered by route panoramas displayed in the traversing window. A region can be visualized with a finite number of local images listed inside the panorama frame, and a block (building, monument, etc.) is snapped in around-object images. The panorama frame has functions of rotating panoramic view, scrolling route panorama, or listing aroundobject images, etc. The map frame is prepared for indicating the location of the current visual node in a global map, and can be swapped with a local map of the visual node. The text window is for detailed description of the node. Another image frame is set for a slide show of discrete images, around-object images, or even a video clip containing dynamic events of the space.

Visual nodes of global and local spaces are organized in a hierarchy (Figure 10a). For example, a district or a town contains many important spots $(0 D)$, routes $(1 D)$ and local regions $(2 D)$, and a region can further contain many buildings $(3 D)$, houses $(2 D)$, sites $(0 D)$ and streets (1D). Links from a global node to local nodes are embedded in the space menu, text and representative local images. The viewer is able to jump to a local visual 

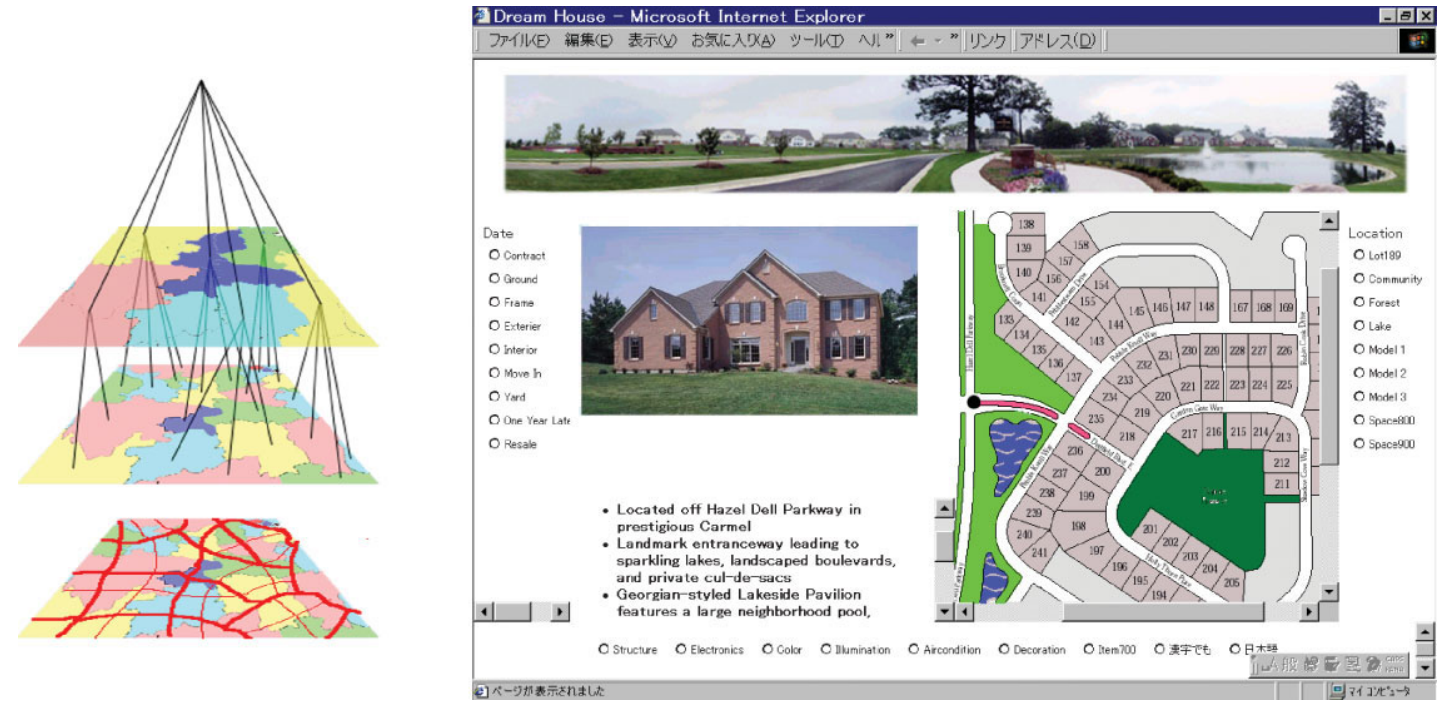

Figure 10. An urban area accessed on the WWW where spaces are indexed from map, view and text list. (a) Hierarchy of visual nodes. (b) Window display of a visual node including various visual information.

node through any of these links. The entire display window will be updated to the data in the selected local node, which realizes an in-depth access. For a global area with many local locations, its visual node includes many representative local views as a general introduction for the panorama frame in the display window.

At the same level of detail, geographically neighbouring spaces are also linked to each other in the map, route panoramas or panoramic views. The viewer can wander around connected visual nodes by clicking the global map or specifying a scene displayed in the image frames. The transition between visual nodes is then realized. At a street crossing, the viewer is able to switch to another street ( $1 D$ to $1 D$ node transition). A transition from a street $(1 D)$ into a building $(2 D)$ is also possible.

\section{Experiments and Applications}

We have succeeded in capturing route panoramas along various streets and in many areas, using vehicles, trains and ships. ${ }^{11}$ The results are continuous, complete and in a compact data format. We are now working on the entire campus of our university and creating a database of route panoramas. The vehicle speed is kept at 20$30 \mathrm{mph}$ approximately in the scene scanning. According to our calculation, the route panorama increases by $6 \mathrm{MB}$ per mile approximately. A web environment has been designed for indexing and virtual navigation in the area.

A broad range of applications can be considered by using our mapped cityscapes in cyberspace. The scenes linked from the map can be used in real estate to find a house or environment in the city. By providing route panoramas, a residential area could be visualized more densely than a series of discrete images. Our modelling and rendering techniques can be used in some historical towns to archive complete scenes faithfully for heritage preservation and exhibition. If we extend the area to an entire city, searching an address on the net will be accompanied by visual information; a visitor will not only find a route to reach an address, but will also be able to follow the visible scenes to the destination. This will enhance many business and culture activities in cyberspace.

\section{Conclusion}

This paper addresses general mapping techniques to project cityscapes to a cyberspace for virtual navigation and visualization. We use various types of mapping, particularly a new image representation-route panorama-in representing the cyber city. We introduce the projection of route panorama, calibration for route panorama acquisition, the generated shape in the route panorama, and the render approach of route panoramas 
on the WWW. A route panorama registers complete route scenes with a seamless format in a small amount of data, which is very useful for indexing and navigation of an entire city in cyberspace. We have transmitted and rendered route panoramas in real time and achieved a virtual tour in an urban area. The route panoramas can even be displayed on portable devices for various digital city applications.

\section{References}

1. Zheng JY, Tsuji S. Panoramic representation of scenes for route understanding. In Proceedings of the 10th International Conference on Pattern Recognition, Vol. 1, 1990; 161-167.

2. Ishiguro H, Yamamoto M, Tsuji S. Omni-directional stereo. IEEE Transactions on Pattern Analysis and Machine Intelligence 1992; 14(2): 257-262.

3. Chen SE, Williams L. Quicktime VR: an image-based approach to virtual environment navigation. In SIGGRAPH95, 1995; 29-38.

4. Zheng JY, Tsuji S. From anorthoscope perception to dynamic vision. In Proceedings of the IEEE International Conference on Robotics and Automation, Vol. 2, May 1990; 1154-1160.

5. Zheng JY, Tsuji S. Panoramic representation for route recognition by a mobile robot. International Journal of Computer Vision 1992; 9(1): 55-76.

6. Zheng JY, Tsuji S. Generating dynamic projection images for scene representation and understanding. Computer Vision and Image Understanding 1998; 72(3): 237-256.

7. Li S, Hayashi A. Robot navigation in outdoor environments by using GPS information and panoramic views. In Proceeding of the IEEE/RSJ International Conference on Intelligent Robots and Systems, 1998; 570-575.

8. Peleg S, Rousso B, Rav-Acha A, Zomet A. Mosaicing on adaptive manifolds. IEEE Transactions on Pattern Analysis and Machine Intelligence 2000; 22(10): 1144-1154.

9. Zhu ZG, Riseman E, Hanson A. Parallel-perspective stereo mosaics. In International Conference on Computer Vision $2001 ; 345-352$.

10. Zheng JY. Digital route panorama. IEEE Multimedia 2003; 10(3): 57-68.

11. http://www.cs.iupui.edu/ jzheng/RP. Route Panorama, examples of route panorama visualized on the Internet, since $1 / 1 / 2003$.

12. Zheng JY. Stabilizing route panorama. In 17th International Conference on Pattern Recognition, Vol. 1, 2004.

13. Gupta R, Hartley RI. Linear pushbroom cameras. IEEE Transaction on Pattern Analysis and Machine Intelligence 1997; 19(9): 963-975.

14. Kawanishi T, Yamazawa K, Iwasa H, Takemura H, Yokoya N. Generation of high-resolution stereo panoramic images by omnidirectional imaging sensor using hexagonal pyramidal mirrors. In 14th International Conference on Pattern Recognition, Vol. 1, 1998; 485-489.
15. Li SG. Qualitative representation of scenes along route. Journal of Image and Vision Computing 17(9): 685-700.

16. Aihara N, Iwasa H, Yokoya N, Takemura H. Memorybased self-localization using omni-directional images. In 14th International Conference on Pattern Recognition 1998; 2: 1799-1803.

\section{Authors'biographies:}

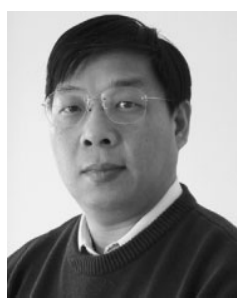

Jiang Yu Zheng received B.S. from Fudan University, China in 1983, and M. S., and PhD degrees in Osaka University in 1987 and 1990, respectively. During 19901993, he was with ATR Telecommunication Research Institute, Japan. During 1994-2000, he was an associate professor in Kyushu Institute of Technology, Japan. Since 2001, he works at the Department of Computer and Information Science, Indiana University Purdue University Indianapolis as an associate professor. His research interests are in computer vision, image processing, virtual reality, and Internet media. He received best paper award from Japan Information Society in 1991 for inventing the first digital panoramic view.

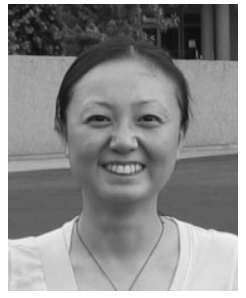

Min Shi received the B.S. and M.E. degrees both in computer science from Zhejiang University, Hangzhou, China, in 1999 and 2002. She is currently a Ph.D student in the Depeartment of Computer and Information Science, Indiana Univerisity Purdue University Indianapolis. Her research interests include 3D computer vision, virtual heritage, image processing and computer art. Her current research is mainly about route panorama, a new digital medium for environment archiving and visualization. 